\title{
HgTe quantum wells with inverted band structure: quantum Hall effect and the large-scale impurity potential
}

\author{
S.V. Gudina ${ }^{1}$, Yu.G. Arapov ${ }^{1}$, V.N. Neverov ${ }^{1}$, S.M. Podgornykh ${ }^{1,2}$, M.R. Popov ${ }^{1}$, \\ E.V. Deriushkina ${ }^{1}$, N.G. Shelushinina ${ }^{1}$, M.V. Yakunin ${ }^{1,2}$, N.N. Mikhailov ${ }^{3}$, \\ and S.A. Dvoretsky ${ }^{3}$ \\ ${ }^{1}$ M.N. Miheev Institute of Metal Physics of Ural Branch of Russian Academy of Sciences \\ 18 S. Kovalevskaya Str., Ekaterinburg 620108, Russia \\ ${ }^{2}$ Ural Federal University, 19 Mira Str., Ekaterinburg 620002, Russia \\ ${ }^{3}$ A.V. Rzhanov Institute of Semiconductor Physics of Siberian Branch of Russian Academy of Sciences \\ 13 Lavrentyev Ave., Novosibirsk 630090, Russia \\ E-mail: svpopova@imp.uran.ru
}

Received December 29, 2018, published online February 25, 2019

\begin{abstract}
We report on the longitudinal and Hall resistivities of a HgTe quantum well with inverted energy spectrum $\left(d_{Q W}=20.3 \mathrm{~nm}\right)$ measured in the quantum Hall $(\mathrm{QH})$ regime at magnetic fields up to $9 \mathrm{~T}$ and temperatures $2.9-50 \mathrm{~K}$. The temperature dependence of the QH plateau-plateau transition (PPT) widths and of variable range hopping (VRH) conduction on the Hall plateaus are analyzed. The data are presented in a genuine scale form both for PPT regions and for VRH regime. Decisive role of the long-range random potential (the potential of remote ionized impurities) in the localization-delocalization processes in the $\mathrm{QH}$ regime for the system under study is revealed.
\end{abstract}

Keywords: mercury telluride, quantum Hall effect, variable range hopping, scaling, localization length, largescale random potential.

\section{Introduction}

A remarkable property of the HgTe-based quantum well (QW) structures is that transitions between band insulator (BI), topological insulator (TI) and semimetal (SM) phases may be achieved by tuning the quantum well thickness $d_{Q W}[1-3]$ (see, for example, Fig. 1 in [2]). The ordinary 2D insulator state is realized at small well widths (up to a critical thickness $d_{c} \approx 6.3 \mathrm{~nm}$ ) [1], while 2D TI exists at larger well widths (up to $d_{Q W} \approx 14 \mathrm{~nm}$ ) [2]. For the width $d_{Q W} \approx 18-20 \mathrm{~nm}$ and wider quantum wells SM 2D state with overlapped conduction and valence $2 \mathrm{D}$ bands is realized [2,3]. A clear model for the physics of the relevant subbands of $\mathrm{HgTe} / \mathrm{CdTe}$ QW based on the bulk HgTe and CdTe band structure is presented in [1].

When $\mathrm{Cd}(\mathrm{Hg}) \mathrm{Te} / \mathrm{HgTe} / \mathrm{Cd}(\mathrm{Hg}) \mathrm{Te}$ structures with $\mathrm{HgTe}$ quantum well (QW) are grown, for a thin QW layer the quantum confinement gives rise to the "normal" sequence of subbands, similar to CdTe, i.e., the bands with primarily $\Gamma 6$ symmetry are the conduction subbands and the $Г 8$ bands contribute to the valence subbands (BI phase).
As the QW thickness is increased, the material looks more like HgTe and for wide QW layers the band structure tends to be "inverted". The inverted regime is achieved when QW width, $d_{Q W}$, exceeds a critical value $d_{C} \cong 6.3 \mathrm{~nm}$. At $d_{Q W} \approx d_{C}$ the conduction and valence bands touch each other that leads to a single-valley gapless 2D Diracfermion system where the quantum Hall effect (QHE) can be observed up to nitrogen temperatures [4].

At the critical thickness $d_{c}$ a topological phase transition from a 2D BI with "normal" band ordering to a 2D TI with an "inverted" one occurs [2]. Recent years demonstrate astounding growth in research on topological insulators, the materials that have a bulk band gap like an ordinary insulator but support conducting states on their edge, socalled quantum spin Hall (QSH) states [5].

The first 2D TIs discovered were based on $\mathrm{HgTe} / \mathrm{Cd}(\mathrm{Hg}) \mathrm{Te}$ quantum wells. The origin of the 2D TI phase in $\mathrm{HgTe} / \mathrm{Cd}(\mathrm{Hg}) \mathrm{Te}$ systems is caused by a peculiar size quantization for HgTe QWs with the inverted band structure [1]. 
The gap between the ground-state heavy hole subband (H1) and the next adjacent subband exists for QW narrower than $18 \mathrm{~nm}$ (for wider wells the QW is in a SM state). While the gap is open, a HgTe-based QW should be a 2D TI having edge states in the gap between subbands. Now the $2 \mathrm{D}$ TI is the most studied and trendy domain for the HgTe-based heterostructures (see, for example, [2,6] and references therein).

In wide $\mathrm{HgTe} / \mathrm{Cd}(\mathrm{Hg}) \mathrm{Te}$ QWs with an inverted energy band structure $\left(d_{Q W} \gtrsim 18 \mathrm{~nm}\right)$ a novel $2 \mathrm{D}$ electron system has been shown to exist: a 2D SM [3,7]. The existence of $2 \mathrm{D} \mathrm{SM}$ in this system is due to the overlap by about a few meV of the conduction band minimum at the center of the Brillouin zone with the side maxima of the valence band. Calculation of the energy band structure [3] shows that a key reason for the overlap in wide QWs is the strain due to the lattice mismatch between HgTe and CdTe.

When the Fermi level crosses both the valence and conduction bands, a number of interesting transport properties caused by the simultaneous presence of 2D electrons and holes have been observed in HgTe QWs.

In SM domain the emphasis is made on a classical magnetoresistance, Shubnikov-de Haas oscillation (SHO) pattern and QHE for two [3] or even three [8,9] types of carriers in a single [3,8] or in a double [9] QW, in weakly doped structures or at different density ratios of the twodimensional electrons and holes in the structures with an applied gate voltage $V_{g}$ [3,8,9].

We present a study of quantum magnetotransport in a 20.3-nm-wide HgTe QW grown on the (013) GaAs substrate, symmetrically modulation doped with In at both sides of QW. Formally, we are in a SM phase but doping with In ensures the Fermi level position in the conduction band above the lateral maximum of the valence band. Because of this we observe an ordinary picture of QHE for one type of carriers (electrons) that allows us to investigate more subtle effects of localization-delocalization in the QHE regime. It is an identification of scaling conditions both for the quantum phase plateau-plateau transition and for the variable-range hopping conductivity on the localized states at the Hall plateaus.

QHE plateau-plateau transition, as well as the plateauinsulator transition, in high-quality HgTe QW with an inverted band structure were first studied and analyzed within scaling concepts at $T=0.3-3.0 \mathrm{~K}$ [10] where it was concluded that the applicability of scaling models to this system is problematic.

In our previous work [11], we have presented the data on the temperature dependence of the PPT width, $v_{0}(T)$, for a HgTe quantum well with inverted energy spectrum $\left(d_{Q W}=20.3 \mathrm{~nm}\right)$. The actual scaling behavior $v_{0}(T) \propto T^{\kappa}$ is observed for the $1 \rightarrow 2 \mathrm{PPT}$ in a wide temperature range $T=2.9-30 \mathrm{~K}$.
Recently, using the scaling approach for the $1 \rightarrow 2$ PPT in non-inverted HgTe QW $\left(d_{Q W}=5.9 \mathrm{~nm}\right)$, Khouri et al. [12] have found an excellent agreement with the universal scaling theory: the scaling coefficient $\kappa=0.45 \pm 0.04$ at $T=0.3-60 \mathrm{~K}$.

As for the variable-range hopping conductivity in the minima of $\sigma_{x x}$, associated with the Hall plateau regions, it is a widely used method for a detection of the localization length divergence in $\mathrm{QHE}$ regime at a number of 2D systems (see, for example, [10-18] in Ref. 13). But for the HgTe-based 2D system, this method was first used by us $[13,14]$ : an analysis of the VRH conductivity in the regions of the first and second quantum Hall plateaus provided an opportunity to determine the value and the magnetic-field dependence of the localization length in the HgCdTe/HgTe/HgCdTe heterostructure with a wide HgTe quantum well.

The objectives of this work are:

- to revisit QHE regime and generalize the data by presenting them in a genuine scale form both for PPT regions and for VRH regime;

— to find more evidences of the scale of random impurity potential in the $\mathrm{QHE}$;

— to dovetail our results on PPT and on VRH with a general picture of studies the localization effects in QHE for systems both with short-range and large-scale random impurity potential.

The analysis has led us to the conclusion that, similarly to modulation-doped GaAs, for PPT in our HgTe QW we are in the intermediate between quantum tunneling and classical percolation region of localization length divergence. On the other hand, the VRH regime in our system is realized by hopping between localized states in the tails of Landau levels, which is within the scope of the laws for classical percolation outside the region of quantum tunneling. All this indicates the decisive role of the long-range random potential (the potential of remote ionized In impurities) for scattering and localization of carriers in the system under study.

\section{Experimental results and discussions}

The sample is a 20.3-nm-wide HgTe quantum well between $\mathrm{Hg}_{0.35} \mathrm{Cd}_{0.65} \mathrm{Te}$ barriers grown on the (013) GaAs substrate, symmetrically modulation doped with In at both sides at distances of about $10 \mathrm{~nm}$ spacers. The electron gas density is $n_{s}=1.5 \cdot 10^{15} \mathrm{~m}^{-2}$ with a mobility of $22 \mathrm{~m}^{2} /(\mathrm{V} \cdot \mathrm{s})$. The sample is in the shape of a Hall bar with Ohmic contacts.

Figure 1 shows the magnetic-field dependences of the longitudinal $\rho_{x x}$ and Hall $\rho_{x y}$ resistivities for the sample under study at $T=2.9 \mathrm{~K}$. We observe the features characteristic of the QHE regime, i.e., the regions of plateaus in the $\rho_{x y}(B)$ dependences $\left(\rho_{x y}=h / i e^{2}\right)$ with rather sharp transitions between them: for $B \geq 2 \mathrm{~T}$, we can see plateaus with numbers $i=4 ; 3 ; 2 ; 1$. 


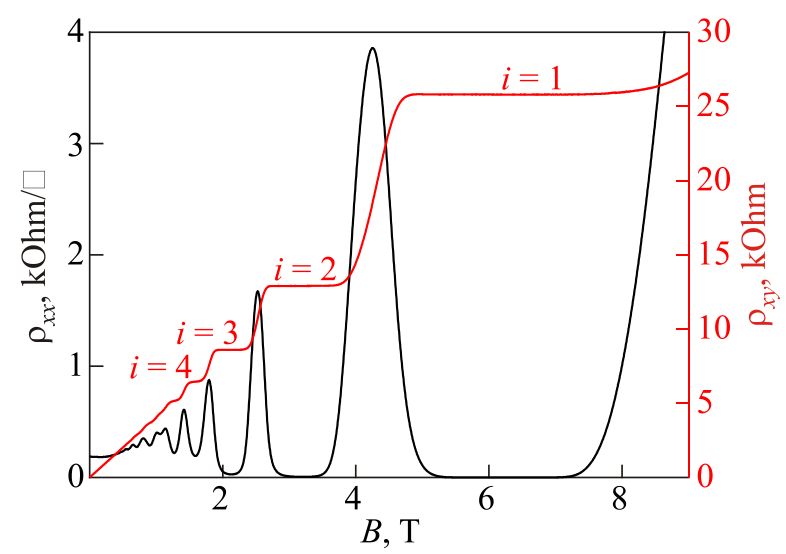

Fig. 1. (Color online) The longitudinal and Hall components of the magnetoresistivity tensor as functions of magnetic field $B$ at $T=2.9 \mathrm{~K}$.

\subsection{Temperature dependences of conductivity in the QHE plateau regions}

The temperature dependences of the $\sigma_{x x}$ at $v=1,2$ and 3 for investigated sample are shown in Fig. 2 as well as the fit of $\sigma_{x x}(T)$ dependences by the Arrhenius equation (straight lines in figure) in the range of more than one (for $v=2$ and 3 ) or even three (for $v=1$ ) orders of magnitude in conductivity at $T \approx 10-50 \mathrm{~K}$.

The extrapolation of $\sigma_{x x}(1 / T)$ dependences to $1 / T \rightarrow 0$ is also demonstrated on Fig. 2. It is seen that the value of $\sigma_{0}=\sigma_{x x}(1 / T \rightarrow 0)$ tends to $\sigma_{0}=e^{2} / h$ for $v=2$ or 3 , and $\sigma_{0}=2 e^{2} / h$ for $v=1$. The prefactor $\sigma_{0}$ of the activated conductivity in the QHE plateau region was studied theoretically by Polyakov and Shklovskii for the of case both a short-range [15] and of a long-range [16] random potentials. It was shown that for a long-range potential the prefactor is universal and at an integer filling factor it is equal to $2 e^{2} / h$, taking into account the contributions to the

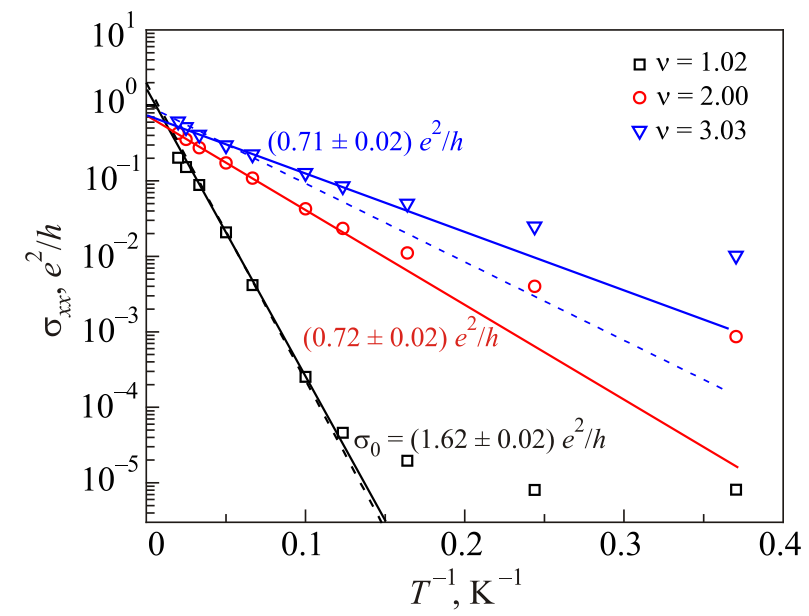

Fig. 2. $\sigma_{x x}(T)$ dependences at $v=1,2$ and 3 with a fitting by Arrhenius equation (straight lines). Values of $\sigma_{0}$ are indicated near straight lines (see text). Dash lines are drawn through the $\sigma_{0}=2$ for $v=1$ and $\sigma_{0}=1$ for $v=3$. conductivity both of electrons on the upper Landau level and of holes on the lower one.

Thus, our results are in good agreement with the work [16], especially for $v=1$. We also emphasize that in our experiment the activation law realized in a rather wide temperature range, as it should be just for the case of a smooth potential (see Fig. 1 in [16]).

Deviations of experimental points from straight lines for $T \leq 10 \mathrm{~K}$ are explained by the variable range hopping among localized states at $E_{F}$, which usually dominates for sufficiently low $T$.

D.G. Polyakov, B.I. Shklovskii and I.L. Aleiner [17-19] showed that in the strongly localized electron system in the QHE-plateau regions, the dominant mechanism of the lowtemperature transport should be the variable-range hopping (VRH) near $E_{F}$ (see also Refs. 20, 21). Consequently, the temperature induced conductivity far from a peak in $\sigma_{x x}(B)$ should be exponentially small. The exponential factor should grow as $E_{F}$ approaches the LL center due to the divergence of the localization length:

$$
\xi \propto\left|B-B_{N}\right|^{-\gamma} \propto\left|v-v_{C}\right|^{-\gamma},
$$

where $B_{N}$ is the value of $B$ at which $E_{F}$ is in the center of $N$ th LL and the critical filling factor $v_{C}$ is a half integer value of $v$. Here $\gamma$ is the critical exponent. The analytical calculation of $\gamma$ is a difficult problem; for the short-ranged impurity potential, numerical methods give $\gamma=2.35 \pm 0.03$ (see, for example, reviews [22,23].

Mott's law describes the VRH for noninteracting 2D electrons [24]:

$$
\sigma_{x x}=\sigma_{0} \exp \left[-\left(T_{0} / T\right)^{1 / 3}\right],
$$

with $k T_{0}=\beta / \xi^{2} g\left(E_{F}\right)$, where $g\left(E_{F}\right)$ is the finite density of states at $E_{F}$ and numerical constant $\beta=13.8 \pm 0.8$ [25].

When the 2D density of states has the form $g(E) \sim\left|E-E_{F}\right|$ and Coulomb repulsion is included the Efros-Shklovskii's (ES) VRH law is being fulfilled [26]

$$
\sigma_{x x}=\sigma_{0} \exp \left[-\left(T_{1} / T\right)^{1 / 2}\right] \text {, }
$$

with $k T_{1}=C e^{2} / 4 \pi \varepsilon \varepsilon_{0} \xi$, determined by the Coulomb energy on the localization length $\xi, C \approx 6.2$ is a numerical constant, $\varepsilon$ is dielectric constant.

A great advantage of VRH method [17-19] is the ability to directly determine the critical index of localization length,

$$
T_{1} \sim 1 / \xi(v) \sim\left|v-v_{c}\right|^{\gamma}
$$

without the mediation of the inelastic collisions exponent, $p$, in contrast to a scaling method for the bandwidth of delocalized states [23].

The concept of VRH conduction proved to be very productive for the interpretation of thermally activated 
transport in the plateau regions of the integer QHE. Direct determination of $\xi$ and its scaling exponent from the ES VRH was done in previous measurements performed on conventional 2DEGs, including Si-MOSFETs, GaAs/AlGaAs and $n$-InGaAs/InAlAs heterojunctions, as well as in the monolayer graphene and in other graphenebased low-dimensional structures.

For GaAs/AlGaAs heterostructures [27] the divergence of localization length with exponent $\gamma=2.3$ was estimated. The results [39] for graphene monolayers should be emphasized: it is the first observation of the crossover from the Efros-Shklovskii's to Mott's VRH for a quantum Hall system, which happens when the localization length exceeds the screening length set by the metallic gate in accordance with the Aleiner and Shklovskii prediction [19].

For HgTe QW, the first study of the temperatureinduced transport at the QHE resistivity minima corresponding to the QHE-plateau regions was done within the concept of variable-range hopping conductivity in our previous works $[13,14]$ on the $\mathrm{HgTe} / \mathrm{HgCdTe}$ system with inverted band structure. Here we returned to this theme summarizing the results obtained and presenting the data in a more universal form.

The longitudinal conductivity $\sigma_{x x}$ as a function of a filling factor at actual QHE minima near $v=1,2$ and 3 is presented in Fig. 3. The activation behavior of $T \sigma_{X X}(T)$ on $(1 / T)^{1 / 2}$ in minima with $v=1$ can be seen in the inset of Fig. 4. Solid lines are the ES law (Eq. (3)) fit of the data.

In Fig. 3 we also show the localization length $\xi(v)$ computed by (Eq. (4)) from the values of $T_{1}(v)$, extracted from ES VRH fits of $\sigma_{X X}(T)$, for continuous values of the filling factor. For comparison, a graph of cyclotron radius, $R_{C}$ (multiplied by ten) dependence on $v$ for different magnetic fields is also shown. The minimal localization length found in the middle of $\mathrm{QH}$ plateaus is $\xi_{\min }=100 \mathrm{~nm}$ for $v=1\left(B_{1}=6.3 \mathrm{~T}\right)$ and $\xi_{\min }=200 \mathrm{~nm}$ for $v=2\left(B_{2}=\right.$ $=3.15 \mathrm{~T})$ and $\xi_{\min }=300 \mathrm{~nm}$ for $v=3\left(B_{3}=2.1 \mathrm{~T}\right)$, which is about ten times larger than $R_{C}$.

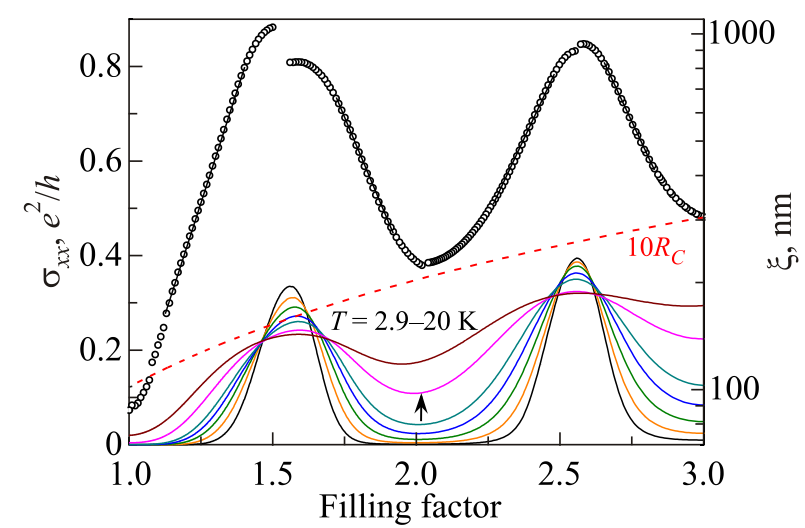

Fig. 3. (Color online) Conductivity $\sigma_{x x}$ in the QHE regime at different temperatures (solid lines) and the localization length $\xi$ (open circles) extracted from ES VRH fits of $\sigma_{x x}(T)$ in comparison with cyclotron radius $R_{C}$ as functions of a filling factor.

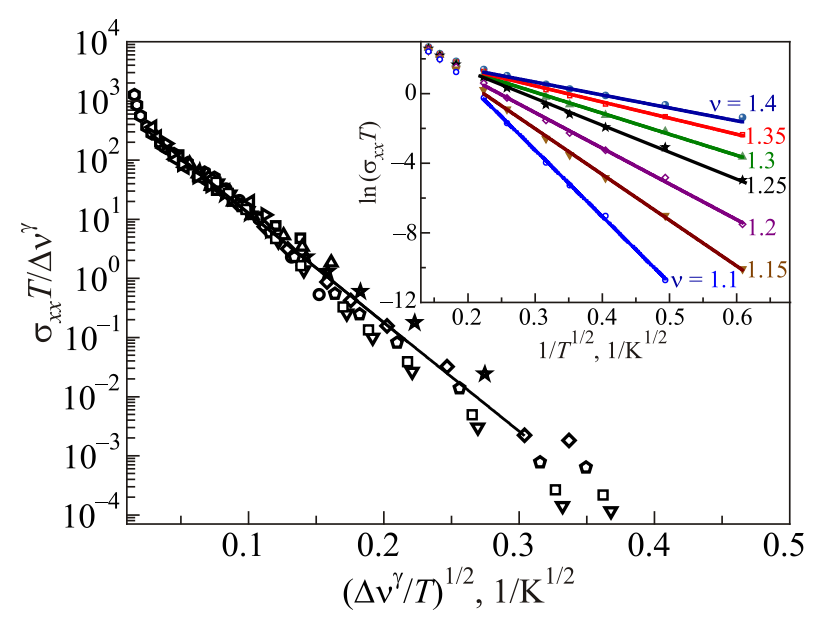

Fig. 4. Conductivity $\sigma_{x x}(T, v)$ as a function of the scale parameter $x=|\Delta v|^{\gamma} / T, \gamma=1.25$ at $v<v_{C}$ for $v_{C}=1.53(T=2.9-15 \mathrm{~K}$, $|\Delta v|<0.35)$. Inset: the log plot of $T \sigma_{x x}(T)$ as a function of $1 / T^{1 / 2}$. Solid lines are the ES law (Eq. (3)) fit of the data.

We emphasize that $\xi_{\min }(v)$ dependence is in correlation just with $R_{C}(v)$ dependence (but not with $l_{B}(v)$ ) in accordance with conclusions of Fogler et. al [29]. The fact that $\xi_{\min } \gg R_{C}$ indicates a large-scale character of the random impurity potential.

To probe the universality of a scaling law in the surrounding area of $v_{c}=1.5$ or 2.5 we have plotted all the conductivity data $\sigma_{x x}(v, T)$ as a function of a single parameter $x=|\Delta v|^{\gamma} / T \sim T_{1} / T$. The values for $\gamma$ are taken from the power-law fits of $T_{1}$ depending on $\left|v-v_{c}\right|$. An example of scaling behavior for conductivity $\sigma_{x x}$ as a function of $x$ at $v_{C}=1.5$ is shown in Fig. 4.

Rescaling the axes shows that all experimental points fall onto straight lines for nearly four orders of conductivity at $|\Delta v| \lesssim 0.35$ both for $v_{c}=1.5$ and 2.5. Thus, Fig. 4 demonstrates the universal single-parameter scaling for the conductivity in the VRH regime at QHE plateaus in analogy with the observations of scaling behavior for VRH in GaAs/ $\mathrm{Al}_{x} \mathrm{Ga}_{1-x} \mathrm{As}$ structure [30,31].

Note that the exponent values $\gamma=1.31 \pm 0.03$ $(0.2 \lesssim|\Delta v| \lesssim 0.33)$ at $v_{C}=1.53$ correlate well with the exact theoretical result $\gamma=4 / 3$, obtained in a theory of classical percolation for systems with large-scale impurity potentials [32].

\subsection{Plateau-plateau transition}

The integer quantum Hall effect regime can be considered as a sequence of insulator-metal-insulator quantum phase transitions when the density of states of a disordered 2D system is scanned by $E_{F}$ in a quantizing magnetic field. We analyze data on the magnetic-field and temperature dependences of conductivity in the regions of the plateauplateau transitions within framework of a scaling hypothesis for a quantum phase transition [23].

Let us concentrate our investigation on the region of the transition between the first and second QHE plateaus and 


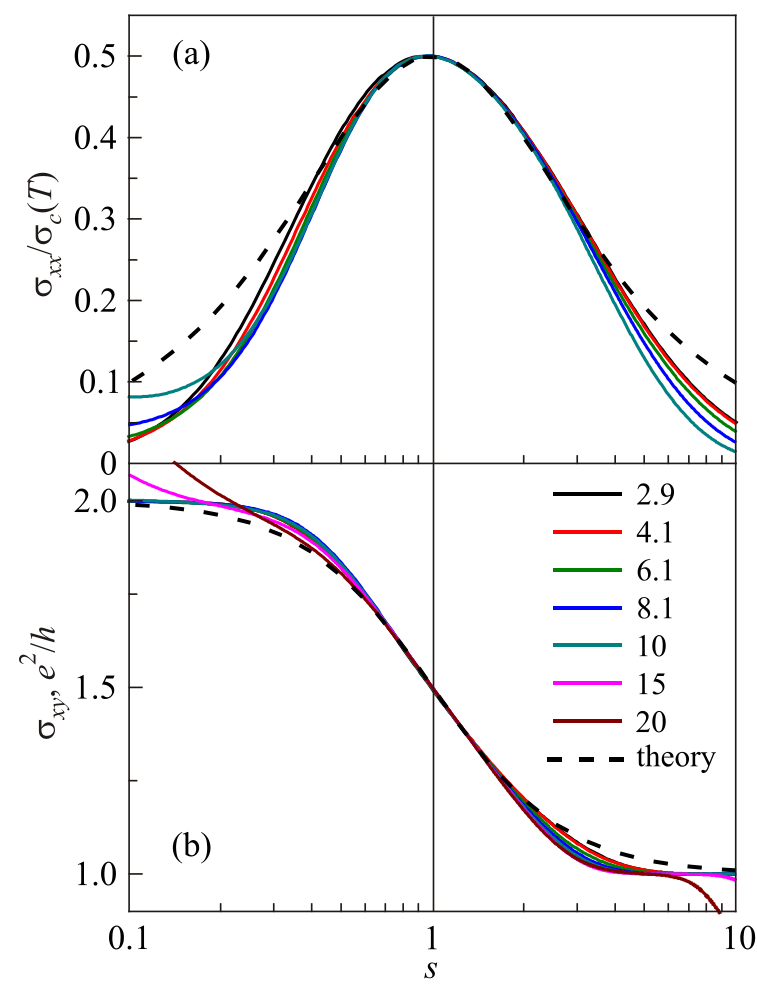

Fig. 5. (Color online) Longitudinal $\sigma_{x x}$ (a) and Hall $\sigma_{x y}$ (b) conductivities as functions of the scaling variable $s$, in the PPT $1 \rightarrow 2$ region at $v_{C}=1.5$. The dashed black lines indicate fitting to Eq. (5).

analyze the temperature dependence of the transition width in the vicinity of the critical magnetic field $\left(B_{C}=4.1 \mathrm{~T}\right)$. For a treatment of the data in a region of $(i-1) \rightarrow i$ PPT, we used interpolation formulas via the so-called scattering parameter (see, e.g., [33] and references therein):

$$
\sigma_{x x}=\frac{s}{1+s^{2}}, \quad \sigma_{x y}=i-\frac{s^{2}}{1+s^{2}},
$$

where $s$ varied from 0 to $\infty$, it is unity at the critical point $v=v_{C}$ and exponentially depends on the filling factor in the vicinity of the critical point:

$$
s=\exp \left(-\frac{\Delta v}{v_{0}(T)}\right)
$$

Here $v_{0}(T)$ is the effective bandwidth of delocalized states at the temperature $T$.

Scaling with a single parameter $s \propto\left|\nu-v_{C}\right| / T^{\mathrm{K}}$ with $\kappa=0.54$ for the dependences both of $\sigma_{x x}(v, T)$ and of $\sigma_{x y}(v, T)$ at $1 \rightarrow 2$ QHE transition is presented on Figs. 5(a), (b). It is seen that a scaling behavior is valid for $0.7 \lesssim s \lesssim$ $\lesssim 2.5$ at $T=2.9-10 \mathrm{~K}$ for $\sigma_{x x}$ and $T=2.9-20 \mathrm{~K}$ for $\sigma_{x y}$.

\section{Discussion of results}

The consideration of the conductivity behavior in the QHE regime as an electron localization-delocalizationlocalization quantum phase transition is the way of theoretical description of this phenomenon, aroused already in the first papers on QHE interpretation [34,35] and allowed to understand its physical nature (see, e.g., the reviews [22,23,36-39]). The theoretical details are well introduced in [22,23,38-40].

A schematic representation of localization length divergences with $\left|v-v_{C}\right|$ is provided in Fig. 6 for a shortrange (inset) and a large-scale impurity potentials according to theoretical considerations [22,23,38-40]. The solid lines in Fig. 6 are: the dependence (1) with $\gamma=\gamma_{p}=4 / 3$ in regions of classical percolation (blue lines) and with $\gamma=$ $=\gamma_{q}$ in regions of the quantum tunneling processes (red lines). Here $\gamma_{q}=7 / 3$ within a modified percolation model [41] and $\gamma_{q}=2.3$ within the modern network model [22,23]. The dash lines in Fig. 6 show an intermediate region of Eq. (1) with $4 / 3<\gamma<7 / 3$ (or 2.3) that gives $0.42<\kappa<0.75$ (with the exponent $p=2$ ) in the interval of crossover from a classical percolation to the quantum tunneling as pointed out by Li et al. [42].

We believe that the critical exponent value for the bandwidth of delocalized states, $\kappa=0.54 \pm 0.01$ obtained by us, as well as a number of results with $\kappa=0.5-0.75$ for systems with large-scale impurity potentials are driven by a situation schematically represented on Fig. 6: the line $L \varphi=$ const crosses the curves $\xi(v)$ just at the intermediate region of $\gamma$ values. This situation, quite possibly, is typical for modulation doped GaAs/AlGaAs heterostructures [40] resulting in "nonuniversal" values of parameter $\kappa$ in the range of 0.5-0.7. Note that for HgTe-based heterostructure with inverted band spectrum $\left(d_{W}=21 \mathrm{~nm}, n=1.5 \cdot 10^{15} \mathrm{~m}^{-2}\right)$ a scaling regime in the QHE has been investigated earlier by Olshanetsky et al. [10] and a lower value, $\kappa=0.49$, was obtained for $1 \rightarrow 2$ PPT at helium temperatures (0.3-3.0) K.

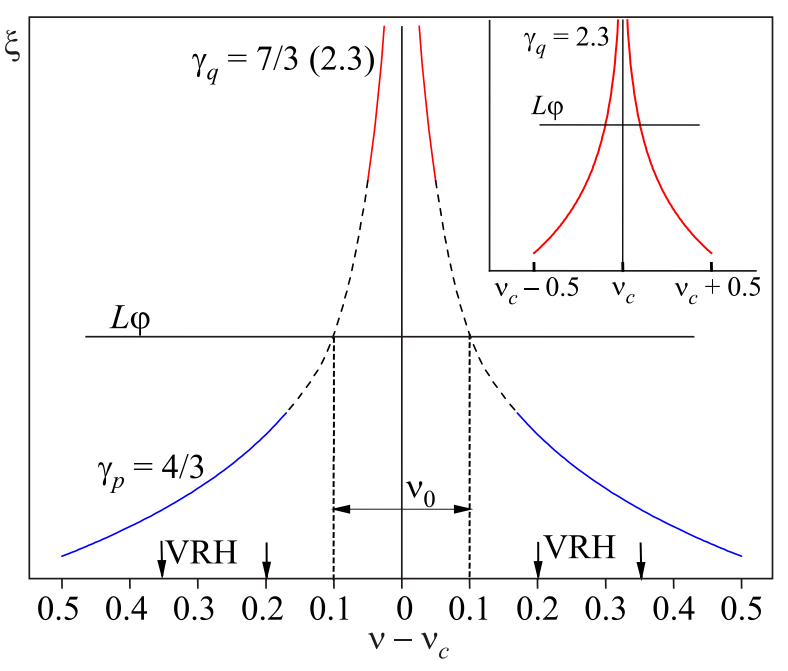

Fig. 6. (Color online) Localization length dependences on the filling factor $v$ within a modern theoretical conception for a largescale impurity potential in QHE regime (see a description in the text). Inset: $\xi(v)$ dependences for a short-range impurity potential. A critical exponent of localization length theoretically is $\xi \approx 2.3$ for $\left|v-v_{c}\right| \leq 0.5$. 
On the other hand, in a recent study [12] on HgTe quantum well with $d_{W}=5.9 \mathrm{~nm}$, that is below critical thickness $d_{c}$, Khouri et al. observed a quantized Hall conductivity up to $60 \mathrm{~K}$ at high carrier concentration $n=4.6 \cdot 10^{15} \mathrm{~m}^{-2}$. From the scaling behavior, realized for the PPT in a wide temperature range $0.3-30 \mathrm{~K}$, they have found the coefficient $\kappa=0.45 \pm 0.04$ for the transition $v=2 \rightarrow 1$ and $\kappa=$ $=0.40 \pm 0.02$ for the $v=3 \rightarrow 2$ transition in excellent agreement with the universal scaling theory for systems described by short-range scattering (see, for example, [23]).

The high carrier concentration [12], achieved by applying gate voltages for a tuning the Fermi energy deep into the conduction band, apparently promotes an effective screening of large-scale potential fluctuations as well as of any inhomogeneities.

As for the variable-range hopping conduction, this mechanism of the low-temperature transport takes place in the regions of localized states at $\xi(E)<L \varphi$. In these regions, localization length $\xi$ and its critical exponent may be determined by a direct way.

For a short-range potential (see inset on Fig. 6) a combination of the values of $\kappa \approx 0.42$ (if $p=2$ ) from an analysis of PPT width at $\xi(E)>L \varphi$ and $\gamma \approx 2.3$ from VRH analysis at $\xi(E)<L \varphi$ should be observed. Just such a situation is, for example, implemented for monolayer graphene $[28,43]$ (except the zero Landau level). Generally, the value of $\gamma \approx 2.3$, which is predicted by a model for a short-range impurity potential [22,23], has been observed in a number of works on VRH (see references in Refs. 24, 25).

On the other hand, in the AlGaAs/GaAs heterostructure (a symmetrical modulation-doped GaAs quantum well bounded by Si $\delta$-doped AlGaAs layers on each side) the values of $\gamma$ are found to be $1.3 \pm 0.2$ with a perfect fit of longitudinal conductivity $\sigma_{x x}$ as a function of the scaling variable for the VRH regions at three filling factors $v=5$, 6 and 7 [31].

These values, which correspond to $\gamma=4 / 3$ in the theories of classical percolation [32,44], show that just classical percolation dominates scaling behavior in the samples due to the presence of the long-range potential fluctuation caused by remote ionized impurities in AlGaAs.

Cobaleda et al. [45] measured the critical exponent in bilayer graphene (encapsulated by $h$-BN) for a number of PPTs, at different carrier densities tuned by the back gate voltage $\left(V_{g}\right)$, both for negative and positive charge carriers. From the analysis of the longitudinal conductivity in the regime of variable range hopping at different $V_{g}$, a set of estimates for $\gamma$ have been obtained with the mean value $\gamma=1.25(0.96,1.54)$. This value is entirely compatible with a classical percolation picture $(\gamma=4 / 3)$ and is definitely different from the value of $\gamma=2.38$, which has been found in monolayer graphene [28].

In our system, the scaling in VRH regime is realized at a sufficiently large distance from the center of the Landau level: for $\left|v-v_{c}\right| \gtrsim 0.2$ (see Fig. 6), displaying the divergence of localization length with the exponents $\gamma=$ $=1.31 \pm 0.03$ at $v_{C}=1.5$. Thus, we deal with the hopping between localized states in the tails of Landau levels, that is within the scope of the laws of classical percolation outside the region of quantum tunneling for a long-ranged impurity potential of the remote In ions.

Let's recall that in the system studied electrons are supplied into the quantum well by indium impurities which are located in delta-layers at both sides of QW at distances of $d \approx 10 \mathrm{~nm}$. It is natural to assume that the Coulomb potential of the remote charged In impurities is the primary source of carrier scattering in the 2D system which corresponds to a smooth random potential for selectively doped systems with a sufficiently large spacer $d$, larger than the magnetic length $\lambda[16,46,47]$.

In our sample, two alternative scaling laws for PPT width $v_{0}(T)$ are valid for different regions of $\Delta v$ : at $\left|v-v_{c}\right| \lesssim 0.1$ and within $0.2 \lesssim\left|v-v_{c}\right| \lesssim 0.35$ for VRH regime, that is indicated in Fig. 6 by the arrows, respectively.

\section{Conclusions}

We have measured the longitudinal and Hall resistivities in the quantum Hall regime at magnetic fields $B$ up to $9 \mathrm{~T}$ and temperatures $T=2.9-50 \mathrm{~K}$ for a HgTe quantum well with inverted energy spectrum $\left(d_{Q W}=20.3 \mathrm{~nm}\right)$ symmetrically $\delta$-doped by In with spacers of $10 \mathrm{~nm}$. Temperature dependence of the plateau-plateau transitions width, $v_{0}(T)$, is studied and the actual scaling behavior $v_{0}(T) \propto T^{\mathrm{K}}$ have been observed for the $1 \rightarrow 2$ plateauplateau transition $\left(v_{C}=1.5\right)$ in a wide temperature range 2.9-30 K. The extracted critical exponent $\kappa=0.54 \pm 0.01$ is in quite good agreement with experimental data for other systems with a large-scale impurity potential.

A set of our experimental data on the temperature dependence of conductance in the minima associated with the Hall plateau regions may be successfully interpreted in terms of the variable-range hopping in the presence of a Coulomb gap. We have found that the hopping conductivity dominates in the regions of both first and second Hall plateaus, thus we used the theory of hopping of interacting electrons to extract, in a straightforward way, the magnetic-field dependence of the localization length, $\xi(B)$. An analysis of the $\xi(B)$ dependence revealed that for the $\mathrm{HgTe}$ quantum well we deal with the hopping between localized states in the tails of Landau levels in the investigated range of fields and temperatures that corresponds to the region of classical percolation through a long-range impurity potential of the remote In ions.

The results we obtained suggest the possibility of implementing the scaling regime both for the QHE plateauplateau transition $\left(\left|v-v_{c}\right| \lesssim 0.1\right)$ and VRH regime within the Hall plateau regions $\left(0.2 \lesssim\left|v-v_{c}\right| \lesssim 0.35\right)$ in the $2 \mathrm{D}$ structures based on mercury telluride. 
We are grateful to B.I. Shklovskii for valuable remarks. Experiments were carried out at the Collaborative Access Center "Testing Center of Nanotechnology and Advanced Materials” of the M.N. Miheev Institute of Metal Physics of the Ural Branch of the Russian Academy of Sciences. The research was carried out within the state assignment of Russian Ministry of Science and High Education (theme "Electron" No. AAAA-A18-118020190098-5) and Complex Program of RAS Ural Branch 18-10-2-6, supported in part by RFBR (projects Nos. 18-02-00172 (samples), 18-32-00382 (experiment) and 18-02-00192 (theoretical support)).

1. M. König, H. Buhmann, L.W. Molenkamp, T. Hughes, C.-X. Liu, X.-L. Qi, and S.-C. Zhang, J. Phys. Soc. Jpn. 77, 031007 (2008).

2. S.S. Krishtopenko, I. Yahniuk, D.B. But, V.I. Gavrilenko, W. Knap, and F. Teppe, Phys. Rev. B 94, 245402 (2016).

3. Z.D. Kvon, E.B. Olshanetsky, E.G. Novik, D.A. Kozlov, N.N. Mikhailov, I.O. Parm, and S.A. Dvoretsky, Phys. Rev. B 83, 193304 (2011).

4. D.A. Kozlov, Z.D. Kvon, N.N. Mikhailov, S.A. Dvoretskii, S. Weishäupl, Y. Krupko, and J.-C. Portal, Appl. Phys. Lett. 105, 132102 (2014).

5. G.M. Gusev, Z.D. Kvon, O.A. Shegai, N.N. Mikhailov, and S.A. Dvoretsky, Solid. State Commun. 205, 4 (2015).

6. K.-M. Dantscher, D.A. Kozlov, M.T. Scherr, S. Gebert, J. Barenfanger, M.V. Durnev, S.A. Tarasenko, V.V. Bel'kov, N.N. Mikhailov, S.A. Dvoretsky, Z.D. Kvon, J. Ziegler, D. Weiss, and S.D. Ganichev, Phys. Rev. B 95, 201103(R) (2017).

7. M. Zholudev, F. Teppe, M. Orlita, C. Consejo, J. Torres, N. Dyakonova, M. Czapkiewicz, J. Wróbel, G. Grabecki, N. Mikhailov, S. Dvoretskii, A. Ikonnikov, K. Spirin, V. Aleshkin, V. Gavrilenko, and W. Knap, Phys. Rev. B 86, 205420 (2012).

8. G.M. Minkov, A.V. Germanenko, O.E. Rut, A.A. Sherstobitov, S.A. Dvoretski, and N.N. Mikhailov, Phys. Rev. B 88, 155306 (2013).

9. M.V. Yakunin, A.V. Suslov, M.R. Popov, E.G. Novik, S.A. Dvoretsky, and N.N. Mikhailov, Phys. Rev. B 93, 085308 (2016).

10. E.B. Olshanetsky, S. Sassine, Z.D. Kvon, N.N. Mikhailov, S. A. Dvoretsky, J.C. Portal, and A.L. Aseev, JETP Lett. 84, 565 (2006).

11. Yu.G. Arapov, S.V. Gudina, V.N. Neverov, S.M. Podgornykh, M.R. Popov, G.I. Harus, N.G. Shelushinina, M.V. Yakunin, N.N. Mikhailov, and S.A. Dvoretsky, Semiconductors 49, 1545 (2015).

12. T. Khouri, M. Bendias, P. Leubner, C. Brüne, H. Buhmann, L.W. Molenkamp, U. Zeitler, N.E. Hussey, and S. Wiedmann, Phys. Rev. B 93, 125308 (2016).

13. Yu.G. Arapov, S.V. Gudina, V.N. Neverov, S.M. Podgornykh, M.R. Popov, G.I. Harus, N.G. Shelushinina, M.V. Yakunin, S.A. Dvoretsky, and N.N. Mikhailov, J. Low Temp. Phys. 185, 665 (2016).
14. S.V. Gudina, Yu.G. Arapov, V.N. Neverov, S.M. Podgornykh, M.R. Popov, N.G. Shelushinina, M.V. Yakunin, S.A. Dvoretsky, and N.N. Mikhailov, Phys. Status Solidi C 13, 473 (2016).

15. D.G. Polyakov and B.I. Shklovskii, Phys. Rev. Lett. 73, 1150 (1994).

16. D.G. Polyakov and B.I. Shklovskii, Phys. Rev. Lett. 74, 150 (1995).

17. D.G. Polyakov and B.I. Shklovskii, Phys. Rev. Lett. 70, 3796 (1993).

18. D.G. Polyakov and B.I. Shklovskii, Phys. Rev. B 48, 11167 (1993).

19. I.L. Aleiner and B.I. Shklovskii, Phys. Rev. B 49, 13721 (1994).

20. B.I. Shklovskii and A.L. Efros, Electronnye svoistva legirovannyh poluprovodnikov, Nauka, Moscow (1979) (in Russian).

21. B.I. Shklovskii and A.L. Efros, Electronic Properties of Doped Semiconductors, Springer, Heidelberg (1984).

22. B. Kramer, T. Ohtsuki and S. Kettemann, Phys. Rep. 417, 211 (2005).

23. B. Huckestein, Rev. Mod. Phys. 67, 367 (1995).

24. N.F. Mott, J. Non-Crystal Solids 1, 1 (1968).

25. A.S. Skal and B.I. Shklovskii, Sov. Fizika Tverdogo Tela 16, 1820 (1976).

26. A.L. Efros and B.I. Shklovskii, J. Phys. C 8, L49 (1975).

27. M. Furlan, Phys. Rev. B 57, 14818 (1998).

28. K. Bennaceur, P. Jacques, F. Portier, P. Roche, and D.C. Glattli, Phys. Rev. B 86, 085433 (2012).

29. M.M. Fogler, A.Yu. Dobin, and B.I. Shklovski, Phys. Rev. B 57, 4614 (1998).

30. Tao Tu, Yong-Jie Zhao, Guo-Ping Guo, Xiao-Jie Hao, and Guang-Can Guo, Phys. Lett. A 368, 108 (2007).

31. Y.J. Zhao, T. Tu, X.J. Hao, G.C. Guo, H.W. Jiang, and G.P. Guo, Phys. Rev. B 78, 233301 (2008).

32. S.A. Trugman, Phys. Rev. B 27, 7539 (1983).

33. P.T. Coleridge, Phys. Rev. B 60, 4493 (1999).

34. H. Aoki and T. Ando, Phys. Rev. Lett. 54, 831 (1985).

35. A.M.M. Pruisken, Phys. Rev. Lett. 61, 1297 (1988).

36. B. Kramer, S. Kettemann, and T. Ohtsuki, Physica E 20, 172 (2003); cond-mat/0309115.

37. V.F. Gantmakher and V.T. Dolgopolov, Phys. Usp. 51, 3 (2008).

38. F. Evers and A.D. Mirlin, Rev. Mod. Phys. 80, 1355 (2008).

39. A.M.M. Pruisken, Int. J. Mod. Phys. B 24, 1895 (2010).

40. S.V. Gudina, A.S. Klepikova, V.N. Neverov, N.G. Shelushinina, and M.V. Yakunin, Fiz. Nizk. Temp. 45, 204 (2019) [Low Temp. Phys. 45, No. 2 (2019)].

41. G.V. Mil'nikov and I.M. Sokolov, JETP Lett. 48, 536 (1989).

42. W. Li, G.A. Csathy, D.C. Tsui, L.N. Pfeiffer, and K.W. West, Phys. Rev. Lett. 94, 206807 (2005).

43. A.J.M. Giesbers, U. Zeitler, L.A. Ponomarenko, R. Yang, K.S. Novoselov, A.K. Geim, and J.C. Maan, Phys. Rev. B 80, 241411(R) (2009).

44. D.-H. Lee, Z. Wang, and S. Kivelson, Phys. Rev. Lett. 70, 4130 (1993). 
45. C. Cobaleda, S. Pezzini, A. Rodriguez, E. Diez, and V. Bellani, Phys. Rev. B 90, 61408(R) (2014).

46. B.I. Shklovskii and A.L. Efros, JETP Lett. 44, 669 (1986).

47. A.L. Efros, Solid State Commun. 65, 1281 (1988).

\section{Квантова яма телуриду ртуті з інвертованою зонної структурою: квантовий ефект Холла та великомасштабний домішковий потенціал}

С.В. Гудина, Ю.Г. Арапов, В.Н. Неверов, С.М. Подгорных, М.Р. Попов, Е.В. Дерюшкина, Н.Г. Шелушинина, М.В. Якунин, Н.Н. Михайлов, С.А. Дворецкий

Експериментально досліджено поздовжній та холлівський опори в квантовій ямі телуриду ртуті з інвертованим зонним спектром $\left(d_{Q W}=20,3\right.$ нм$)$, що виміряні в режимі квантового ефекту Холла (КЕХ) в магнітних полях до 9 Тл та інтервалі температур 2,9-50 К. Проаналізовано температурні залежності ширини переходу між плато КЕХ та провідність зі змінною довжиною стрибка в області плато КЕХ. Дані представлено в універсальній скейлінговій формі як в області переходу між плато, так і в режимі стрибкової провідності. Виявлено вирішальну роль великомасштабного випадкового потенціалу (віддалене легування через спейсер) в процесах локалізаціїделокализации носіїв заряду в режимі КЕХ в дослідженій системі.

Ключевые слова: телурид ртуті, квантовий ефект Холла, стрибкова провідність, скейлинг, довжина локалізації, великомасштабний випадковий потенціал.
Квантовая яма теллурида ртути с инвертированной зонной структурой: квантовый эффрект Холла и крупномасштабный примесный потенциал

\section{С.В. Гудина, Ю.Г. Арапов, В.Н. Неверов, С.М. Подгорных, М.Р. Попов, Е.В. Дерюшкина, Н.Г. Шелушинина, М.В. Якунин, Н.Н. Михайлов, С.А. Дворецкий}

Экспериментально исследованы продольное и холловское сопротивления в квантовой яме теллурида ртути с инвертированным зонным спектром $\left(d_{Q W}=20,3\right.$ нм), измеренные в режиме квантового эффекта Холла (КЭХ) в магнитных полях до 9 Тл и интервале температур 2,9-50 К. Проанализированы температурные зависимости ширины перехода между плато КЭХ и проводимость с переменной длиной прыжка в области плато КЭХ. Данные представлены в универсальной скейлинговой форме как в области перехода между плато, так и в режиме прыжковой проводимости. Выявлена решающая роль крупномасштабного случайного потенциала (удаленное легирование через спейсер) в процессах локализации - делокализации носителей заряда в режиме КЭХ в исследованной системе.

Ключевые слова: теллурид ртути, квантовый эффект Холла, прыжковая проводимость, скейлинг, длина локализации, крупномасштабный случайный потенциал. 